\title{
A further study of alternation of normal and distorted vision ${ }^{1}$
}

\author{
JOAN E. FOLEY \\ UNIVERSITY OF TORONTO
}

Subjects responded in accordance with the apparent distance of a target, alternating between normal vision and minification. The training procedure of Foley \& Abel (1967) was modified in an attempt to facilitate appearance of a phenomenon predicted by Taylor (1962). His expectation that effects and aftereffects would decline with repetition of the changes in visual condition was again not confirmed.

Foley \& Abel (1967) measured the magnitude of effect and aftereffect in Ss required to perform a motor task on alternate sessions with normal vision and while wearing minifying spectacles. The task was to operate a plunger and drive a ball up an inclined plane to a target which could vary in distance. The results failed to support the prediction of Taylor (1962) that the magnitude of effect and aftereffect would diminish with repeated change of visual condition. In view of the null result, it was decided to conduct a further experiment with some changes in procedure in an attempt to produce the phenomenon. Method

The task and method of scoring was as described by Foley and Abel with the following noteworthy changes: (1) Fifteen sessions were conducted in succession on each of nine daily visit to the laboratory (135 sessions). A session comprised five trials without knowledge of results (NKR) followed by 10 trials with knowledge of results (KR). (2) There were four groups of eight Ss each. Two began the task with normal vision (N) and two with glasses (G). In each case one group experienced the normal condition and minification on alternate sessions (as in Foley and Abel), and one on alternate blocks of five sessions. The groups will be designated as $1 \mathrm{~N}, 1 \mathrm{G}, 5 \mathrm{~N}$, and $5 \mathrm{G}$.

These changes were aimed at overcoming possible deficiencies acknowledged by Foley and Abel, specifically, that the distribution and amount of training may not have been favorable to producing the phenomenon.

Performance was measured only where successive sessions were conducted without change of target distance (70 in.) The distribution of "dummy" targets was such as to yield one daily measure of effect and of aftereffect in Groups $5 \mathrm{~N}$ and 5G and three of each type of score daily in Groups $1 \mathrm{~N}$ and 1G. In addition three daily retention scores were available in the case of Groups $5 \mathrm{~N}$ and $5 \mathrm{G}$. These are obtained where successive sessions occur without change of target or of visual condition. The schedule was such that alternate days yielded measures of retention under each visual condition in turn.

\section{Results}

One score obtained was the difference between the mean error on the five NKR trials and that displayed on KR trials in the preceding session $\left(\mathrm{NKR}_{\mathrm{n}}-\mathrm{KR}_{\mathrm{n}-1}\right)$. Analysis of variance of these scores indicated that Ss are susceptible to the changes in visual condition $(F=288.78$, df $=1 / 476, p<.001)$. Groups started with glasses are more susceptible than those started with normal vision $(F=10.04, d f=1 / 476, p<.001)$ and groups on Schedule 5 show more susceptibility than those on Schedule $1(F=7.37, \mathrm{df}=1 / 476, \mathrm{p}<.01)$. However, the magnitude of effect and aftereffect does not change with training $(F<1, d f=8 / 476)$.

The other score is the difference in mean error on $\mathrm{KR}$.trials of successive sessions. Analysis of variance of $K R_{n}-K R_{n-1}$ scores indicated that effect and aftereffect measures still differ ( $F=73.50$, df $=1 / 476, p<.001)$. There is no change in this difference over days $(F=1.58, \mathrm{df}=8 / 476, \mathrm{p}>.05)$. However, adaptation does occur during the knowledge period, although it is not complete. The mean difference between effect and aftereffect scores was calculated for each $S$ from both $N K R_{n}-K R_{n-1}$ and $K R_{n}-K R_{n-1}$ scores over the whole experiment. In all but two of the 32 Ss the latter yielded a smaller difference. The reduction was significant at the .0005 level $(t=$ 4.56, $\mathrm{df}=31$, one-tailed test).

In Groups $5 \mathrm{~N}$ and $5 \mathrm{G}$ the retention scores were used as a baseline for comparison of magnitude of effect and aftereffect. No significant difference in magnitude was found.

Data were available from an additional two groups of eight Ss run under Conditions $5 \mathrm{~N}$ and $5 \mathrm{G}$, respectively, but for only five days (75 sessions). Analysis of $N K R_{n}-K R_{n-1}$ scores showed susceptibility to the visual manipulation $(F=85.54, \mathrm{df}=1 / 126, \mathrm{p}<.001)$ which did not change with training $(F<1)$ and did not depend on starting condition. Introduction of knowledge reduced the effect and aftereffect to insignificant proportions. There was no difference in magnitude of effect and aftereffect.

\section{Discussion}

No confirmation of Taylor's prediction is obtained in spite of the increased opportunity for consolidation of performance within the daily visit to the laboratory and within blocks of sessions in Groups $5 \mathrm{G}$ and $5 \mathrm{~N}$. Response-produced feedback remained 
necessiary for correction of errors induced by changes in visual condition throughout the experiment.

The larger aftereffect reported by Foley and Abel failed to reappear in the present study.

Susceptibility to the changes in visual condition, however, does appear to depend on the schedule of alternation and on the condition under which the novel situation is first experienced. Ss started with glasses by Foley and Abel were more susceptible also, but the difference between groups was not significant in that study. Nor was it in the five-day study described above, although the direction of the difference was the same. The effect is therefore a weak one, but has theoretical implications.

\section{References}

FOLEY, JOAN E., \& ABEL, SHARON M. A study of alternation of normal and distorted vision. Canad. J. Psychol, 1967, 21, 220-230. TAYLOR, J. G. The behavioral basis of perception. New Haven, Yale University Press, 1962.

Note

1. This study was supported by DRB Research Grant No. 9425-15 and NRC Grant APB 1. 